(C)2018 IEEE Personal use of this material is permitted. Permission from IEEE must be obtained for all other uses, in any current or future media, including reprinting/republishing this material for advertising or promotional purposes, creating new collective works, for resale or redistribution to servers or lists, or reuse of any copyrighted component of this work in other works. 


\title{
An inverse perspective mapping approach using monocular camera of Pepper humanoid robot to determine the position of other moving robot in plane
}

\author{
M. Hassan Tanveer ${ }^{1}$ and Antonio Sgorbissa ${ }^{1}$ \\ Department of Bio-Robotics and Intelligent systems (DIBRIS), University of Genova, 16145 Genova, Italy \\ muhammadhassan.tanveer@dibris.unige.it,antonio.sgorbissa@unige.it
}

Keywords: $\quad$ Pepper Humanoid Robot, Inverse Perspective Mapping, Monocular Camera, Moving robot

\begin{abstract}
This article presents a method to know the position of object or moving robot in the plane while the camera is moving dynamically. An Inverse Perspective mapping (IPM) approach has been embedded in a monocular camera on Head of Pepper Humanoid Robot (Softbank Robotics) for real time position determination of other object or robot in plane. While the Pepper head is moving, it is difficult to determine position or distance to objects in front of the robot with any different degree of certainity. By applying IPM, a linear relationship between the IPM frame and world frame becomes the key element to know the position of object while the head is static but when the head orientation changes the IPM is modified to adapt the linear relationship between both frames. So, the proposed method is based on the extraction of accurate bird's-eye view. The method includes the Image Acquistion, IPM Filtering, Detection Phase, Region of Interest Selection and Pixel remapping.
\end{abstract}

\section{INTRODUCTION}

One of the core elements in field robotic research is to implement the social robot's algorithm, that help robots to anticipate the desired task which results the ease for humans. In the twentieth century, the process took place and steps have been put forward for the realization of fully autonomous humanoid robots ( $\mathrm{Si}$ ciliano and Khatib, 2016). In this domain of robotics, the problem of position estimation and distance determination has been one of the concered domain because it can overcome the destruction in robots due to collision (Lemaignan et al., 2017).

Collision detection systems can provide robots with an alert prior to a collision that allows robot to take preventive actions (Stein et al., 2017). The robot processes can be included in the security system as mentioned in (Maurino et al., 2017) which immediately stops the robot processes if an imminent object is detected in surroundings nearby. In addition, determining distance within robots, could also allows the robot to provide alert and to make cautiously comments to the user (Rubenstein et al., 2014). Instructions may move to prevent this problem, or create a security setting for sudden collision (Mukhtar et al., 2015). Many distance determination activities have been carried out using functional systems such as optical cam-

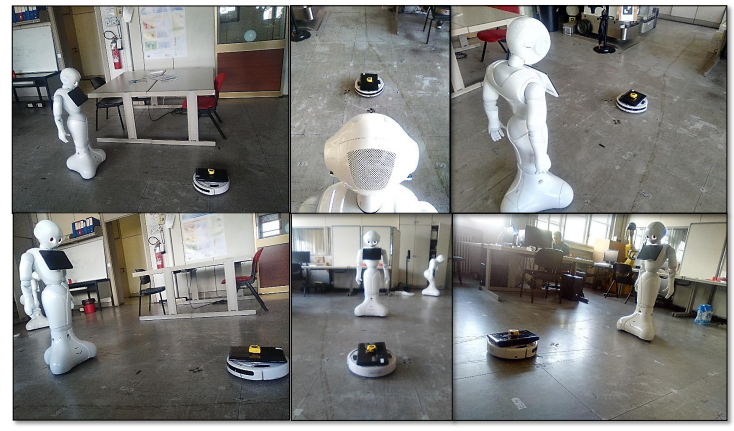

Figure 1: Pepper Tracking other robot in plane

eras that contributes to greater power and intervention in comparison with optical camera sensors (Hiremath et al., 2014). For a very simple idea it is used: Signals used from antenna indicate the purpose of the target goal. Distance and tags can be calculated depending on the length of travel and destination.

A robot should detect the relative position of another robot, while the other robot is in the floor. A large amount of research has been done for distance determination to an object using forward facing cameras (Tuohy et al., 2010), sonar and laser scanners that 
could not be specifically used if the height of other robot is too low. A multi-camera setup as employed by (Ma et al., 2014), provides depth information by establishing feature correspondence and performing triangulation. However, it also carries severe processing and configuration overheads, which is cheaper and is not required on power robots.

In this article, openCV tools has been used to make a blob of color so the specific color object can be detected by camera and term as a potential object. Concurrently, two robots has been adopted Pepper Humanoid robot and iRobot Roomba. The Pepper robot has capabilities for vision which include facial and shape recognition (in-built cameras). Secondly, the iRobot Roomba is a unicycle mobile robot that performs a wide range of task from autonomous cleaning and surveillance, to transportation and assistance to the elderly. By combining both of these robots with potential object, a solution that consists in the composition of a IPM view (bird's eye view) of Pepper head camera has been proposed. This view will serve as a virtual map for the iRobot Roomba in the Pepper Frame of view (FOV) to help the user to adequately specify the commands to be sent to the other Roomba under its control, as in Fig. 1 that is referred to Pepper Frame of view. The proposed system consists of a single forward facing Pepper camera, capturing video images at 30 frames per second (fps).

Section II of this paper discusses the Methodology for distance determination that includes Inverse Perspective Mapping which allows the image perspective to be linearised, the surface subtraction requirement for object detection and the calibration issues. The technological platform with discussion of results are described in section III. Future work concludes the paper in section IV.

\section{METHODOLOGY}

The workflow for the process of calculating object position in moving Pepper Head mainly includes three parts: IPM Filtering: (Image Acquisition, Grayscale conversion and Camera Parameter definition), Detection Phase:(ROI selection and Pixel Remap) and Calculation steps.

The transformation of the coordination system can be done in a mathematical way using the inverse perspective mapping (IPM) that converts picture coordinates from one point to another (Jeong and Kim, 2016). The final homogeneous distribution of the two-dimensional image (input) information in the pixels changes mainly through the IPM-based removal perspective effect.
Objectively, IPM transformation requires a special acquisition conditions (i.e:camera location, orientation, optics, etc.) (Laganiere, 2000) (Lin and Wang, 2012) and some of the assumptions in which the image is presented(here defined as a priori knowledge). Therefore, the IPM transform can be used in a structural environment in which, for example, the camera is placed in a static position or in situations where the caliber system and caliber can be obtained from another type of sensor (Yenikaya et al., 2013) (Guo et al., 2014) (Civera et al., 2008).

In this case, we use the IPM to get a Pepper robot top-down view from the camera. This change thus eliminates the non-linearity of the distances between the frame and the object in the world. Using IPM, the aim is to map pixel points $(u, v)$ to world coordinate points $\left(X_{w}, Y_{w}, Z_{w}\right)$, as in Eq. 1 . The requirement of a rotation about $\theta$, a translation along the cameras optical axis, and a scaling by the camera parameter matrix (Oliveira et al., 2015), can be expressed as:

$$
\begin{gathered}
(u, v, 1)^{T}=\operatorname{KTR}(x, y, z, 1)^{T} \\
R=\left[\begin{array}{cccc}
1 & 0 & 0 & 0 \\
0 & \cos \theta & -\sin \theta & 0 \\
0 & \sin \theta & \cos \theta & 0 \\
0 & 0 & 0 & 1
\end{array}\right] \\
T=\left[\begin{array}{lllcl}
1 & 0 & 0 & 0 \\
0 & 1 & 0 & 0 \\
0 & 0 & 1 & -\frac{h}{\sin \theta} \\
0 & 0 & 0 & 1
\end{array}\right]
\end{gathered}
$$

The intrinsic parameters of the camera are represented by the following matrix:

$$
K=\left[\begin{array}{cccc}
f * k u & s & u_{0} & 0 \\
0 & f * k v & v_{0} & 0 \\
0 & 0 & 1 & 0
\end{array}\right]
$$

where $h$ is the height of camera, $f$ correspond to the focal length measured in horizontal and vertical pixel units $k_{u}$ and $k_{v}$ respectively. The positions $u_{0}, v_{0}$ are the principal points where the optical axis fixes the image plane.

The camera makes a projection of the 3D view point in the world with a picture located on a retina plane. By using a homogeneous coordinate, the projective connection between 3D light and its image points can be changed as:

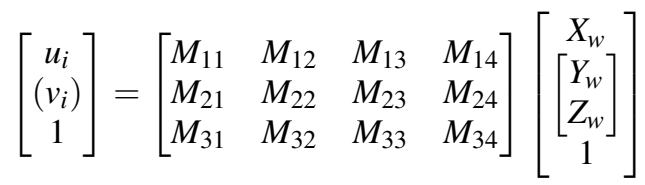


The $3 * 4$ matrix $M_{i, j}$ is the transformation matrix in Eq. 5. It relates world points to image points according to the camera location with respect to the reference frame, represented by a camera matrix K Eq. 4, rotation matrix R Eq. 2 and a translation vector T Eq. 3. If the structure under observation is a plane, it may be simpler to create because the global coordination system can be anywhere, it can be conveniently located on the plane so it has zero coordinate line $Z$. This choice would reduce the projection matrix to:

$$
\left[\begin{array}{c}
u_{i} \\
\left(v_{i}\right) \\
1
\end{array}\right]=\left[\begin{array}{lll}
M_{11} & M_{12} & M_{13} \\
M_{21} & M_{22} & M_{23} \\
M_{31} & M_{32} & M_{33}
\end{array}\right]\left[\begin{array}{c}
X_{w} \\
\left(Y_{w}\right) \\
1
\end{array}\right]
$$

The updated change focuses on a plane of the world into the points of the image as well as a source image may be rewritten as:

$$
\begin{aligned}
& d s t(u, v)=\operatorname{src}\left(\frac{M_{11} X_{W}+M_{12} Y_{W}+M_{13}}{M_{31} X_{W}+M_{32} Y_{W}+M_{33}},\right. \\
& \left.\frac{M_{21} X_{W}+M_{22} Y_{W}+M_{23}}{M_{31} X_{W}+M_{32} Y_{W}+M_{33}}\right)
\end{aligned}
$$

The sampling rate in $X$ direction at this point is simply the number of source image pixels between $(X, Y, 1)^{T}$ and the point $\left(X+(\delta X, Y, 1)^{T}\right)$ divided by the distance between these two points. The transformation depends upon the Eq. 6 and can be warped to its $d s t$ coordinates as mentioned in Eq. 7 .

In detection phase, there are conventional techniques that needs the prior knowledge of the object for detecting it with a camera (Wang, 2013). However, a more effective approach is used to adopt an algorithm for Surface Subtraction (SS). The object to be detected is green in color and wheeled robot has a small green object on top, and thus the surface subtraction algorithm starts from the detection of green object. Considering the scene in Fig. 2, the object we must detect is deemed to be the nearest part of the object in image that is in surface and directly in front of the Pepper. Therefore the easiest way to accurately detect the object is by accurately setting the BGR points of potential object, as stated in steps below:

1. Green channel is separated to its constituent in the BGR image.

2. Taking Green channel into account, the algorithm calculates the average pixel values in $\mathrm{x}$ and $\mathrm{y}$ axes in the captured Pepper image.

3. The algorithm makes a rectangle over the green object and place a red dot at the bottom center of the rectangle to calculate the exact pixel value which is near to the Pepper.
4. The algorithm repeats steps 2 and 3.

From the above mentioned steps the adaptation is easy under various brightness and surface conditions. By using the OpenCV morphology tool we get the green object found under different conditions in light, because the BGR channel range can be solved manually. Therefore, under the conditions of light, the result will remain similar to the presence of a green color due to the upper and lower limits of the BGR morphological tool. It can help identify potential objects by recognizing their colors in varying sizes so that there will be no disruptions under various lighting conditions.

Before we calculate the distance between object and Pepper, we need to calculate the height of the pixel object from below the image, as done in Fig. 2. From the bottom of the binary image in each RGB color space, we calculate the average pixel intensity on each pixel line in the image. From this we find out how high the object lies in the world frame. The ground just before Pepper is considered. This is to minimize the ease of knowing the wrong positives caused by other things within the Pepper frame.

In previous steps, the pixel points of the potential object measured by the image are known, but to calculate it according to the global framework, the calibration tool should be a reasonable unit of measurement (i.e there is a lot of calibration methods, such as (Boyle et al., 2000) (Munaro et al., 2016).

The methods like chessboard calibration and marking points calibration discussed in (Miraldo and Araujo, 2013), (Moreno and Taubin, 2012) can be used as well but in this case, the proposed system would be implemented with a Pepper camera in a fixed or moving position. The placement of a green, blue or red color box with known distance (i.e: known $\mathrm{x}$ and $\mathrm{y}$ axes distance) in the FOV of the camera, along a flat surface, would allow for the measurement of the number of pixels equivalent to distance. Using this value, the number of pixels in the image could be converted to a real world value in meters.

\section{RESULTS AND DISCUSSION}

The algorithm presented here is implemented to Pepper Robot in real time to know the position of other object in plane. For this purpose, the Python language, complemented by the OpenCV library of functions was chosen for implementation that allows the rapid development of image processing algorithms without the need to develop complex algorithms by using its open source library of functions (Van der Walt et al., 2014). 
With Pepper robot, a database of video samples was captured at 30 frames per second (fps), at a resolution of $320 * 240$ pixels. Considering that the change in distance of potential objects is relatively slow compared to the full frame rate of the system, a slower frame rate was considered for the purposes of calculating distance and computing position. Using a sampling rate of every 10 frames produced smooth and reliable results. A frame rate of 10 per second was chosen as it provides a good trade-off between computation time and number of calculations per second.

The first experiment has been done by putting a green object at one point in the Frame of view (FOV) of Pepper Head camera. First it is placed at $0.3 m$ on $X_{\text {axis }}$ and $1.8 \mathrm{~m}$ on $Y_{\text {axis }}$, then the data have been recorded for 300 samples. Then the Pepper head changes its orientation in different Yaw and Pitch as shown in Fig. 2. The same acquisition has been performed to other different points, it can be seen from Figs. 5, 6 that is actual calculation of one point along $\mathrm{x}$ and $\mathrm{y}$ axis. The deviation at different Pitch and Yaw angles are presented in same figure.

It can be seen in bird's-view image, Fig. 2, that the relationship between the potential object and its distance from the camera is linear in nature. However, the change in the position of the pixel of object will proportionally reflect this difference in distance. In order to determine the position of potential object in bird eye view, the calibration with different distance on pixels is considered and sum up in a formula as in Eq. 8 and average error in Eq. 9. It goes something like: we first calibrate the world points in Bird eye view frame with a known distance of object. Then the object is being placed at some distance from Pepper camera and by measuring at the same time the pixel (in bird's-view) corresponding to the object detection. The apparent pixels is measured corresponding to the detected object.

$$
i_{n+1}=o+p_{n}+i_{n}+\text { noise }
$$

where $i$ is frame point, $o$ is the offset, $p$ is the pixel.

$$
\left|e_{k}\right|=\sqrt{\left(x_{\text {odometry }}-x_{I P M}\right)^{2}+\left(y_{\text {odometry }}-y_{I P M}\right)^{2}}
$$

where $x_{\text {odometry }}, y_{\text {odometry }}$ are the reference odometry points in world frame and $x_{I P M}, y_{I P M}$ are the achieved points. The average error and standard deviation of average error are calculated and denoted by $a v|e k|$ and std $|e k|$.

The image has been taken with different angles of Pitch and Yaw by putting the object at one static position. The clearity of IPM view can be seen in Fig.
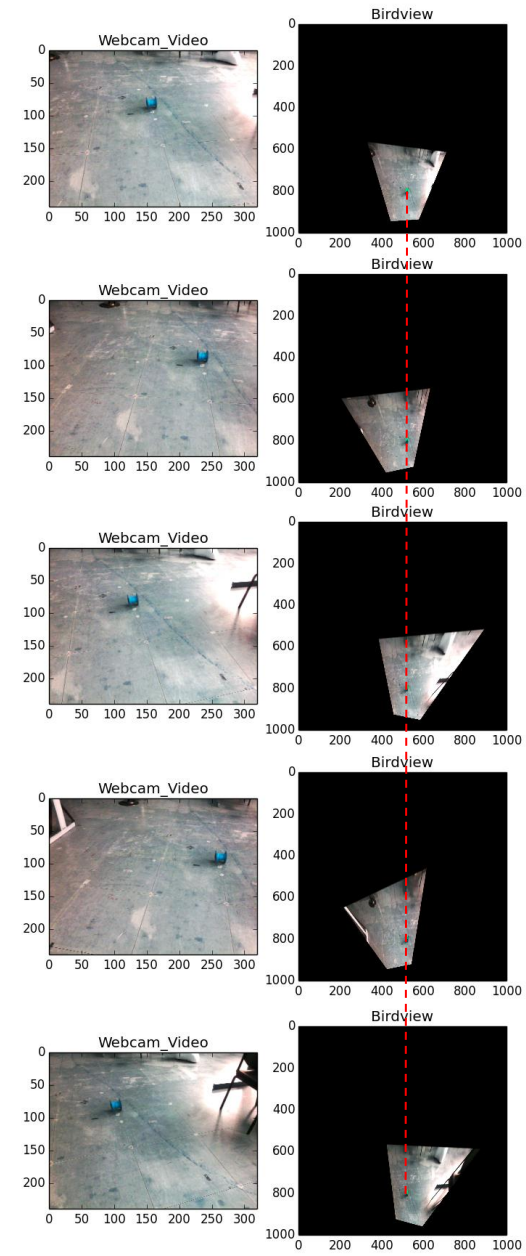

Figure 2: Fixed object IPM view with different Pepper Head Orientation (i.e: $($ yaw,pitch $)=(36,0),(36,-10),(36,10),(36,-$ $20),(36,20))$

2 that due to proposed method the object always implies the same position in IPM view while the camera is moving. The linear straight red line in Fig. 2 shows that the head moving has no impact on the position of object in IPM frame, so it can be converted next to its respective world frame value.

So, for covering the whole plane region of $3.6 * 3.6 \mathrm{~m}$ area, in order to know the object position a total number of 36 points with $0.6 \mathrm{~m}$ apart in both axis has been considered. The red star point in Fig. 3 is the actual point of the floor and the blue dot is the average error of all points. The standard deviation of average error is expressed by error bar of $\mathrm{x}$ and $\mathrm{y}$. The number below each red dot is the number of acquisitions performed with different values of pitch and yaw.

Specifically the next test is being done by putting the object $0.6 \mathrm{~m}$ apart in $X_{\text {axis }}$ and $Y_{\text {axis }}$ direction to check 


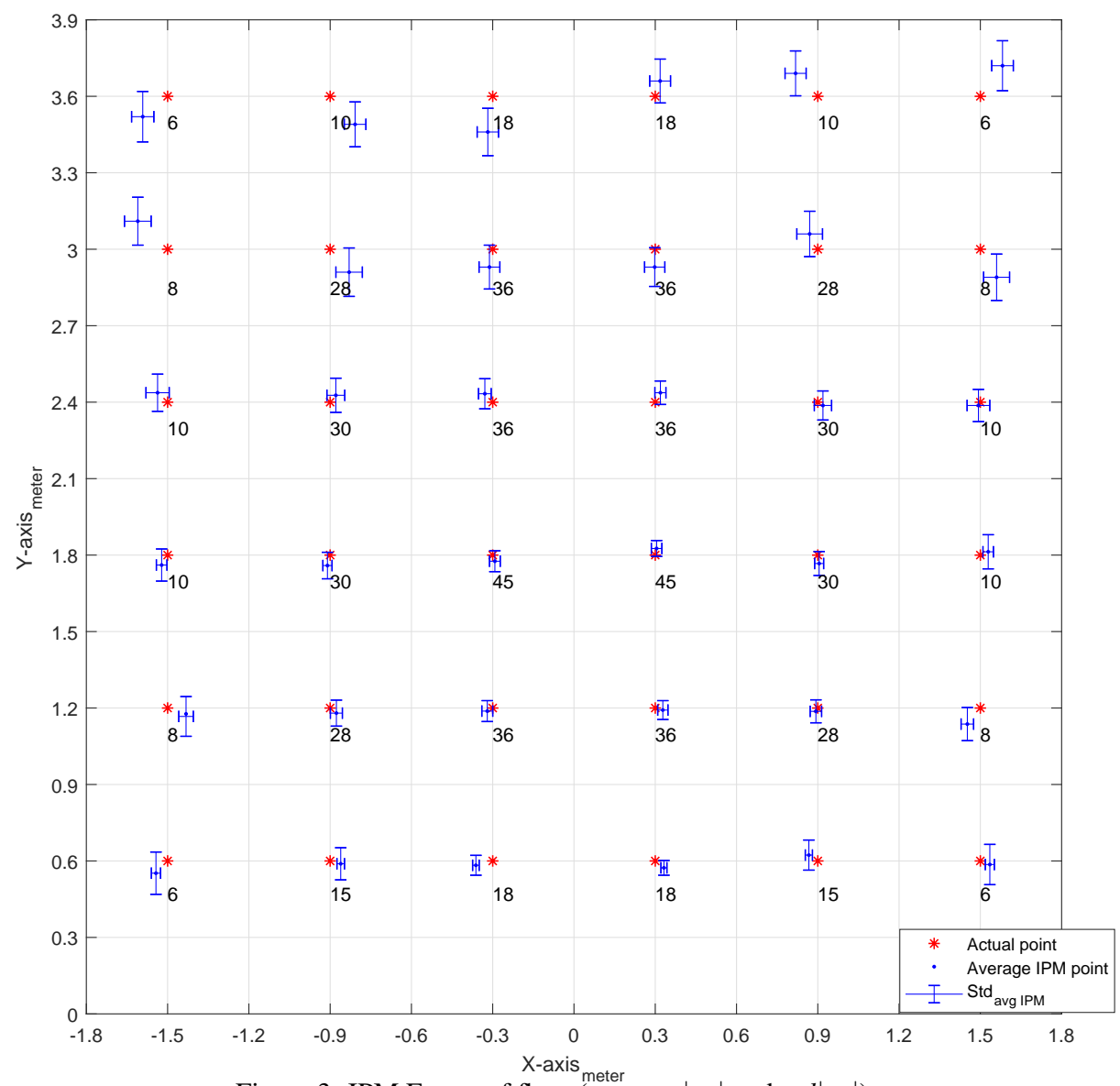

Figure 3: IPM Frame of floor (average $|a v|$ and std $|a v|$ )

the effective of algorithm in far points. The results of all points are shown in Fig. 3. The data recording of each camera angle is being done in Pitch $=36$ to 15 and Yaw $=-20$ to 20 with 5 intervals each that in total becomes 45 values for calculating one point in the best case. The more the object is closer to the centre of the area, the more will be the number of recording, since the object will be always in the FOV of the camera, with all different values of pitch and yaw. On the other side, when the object is closer to the border of the considered area, it will be detected by the camera only with a limited set of pitch-yaw values, thus having a greater number of recording gives less the error deviation. The region in between $1.2 \mathrm{~m}$ to $3 \mathrm{~m}$ in $Y_{\text {axis }}$ (i.e: Pitch Movement) has got almost maximum number of camera view points which gives the less error rate. The values which are far away from $3 \mathrm{~m}$, they give more deviation because the far the object goes the Monocular camera becomes blur which becomes hard to detect the potential object, but still it is detecting with low number of Camera point values of Yaw and Pitch. In a similar manner, the deviation in $X_{a x i s}$ is increasing if it rotate Head more than $-1.2 \mathrm{~m}$ to $1.2 \mathrm{~m}$ in Yaw Orientation. Apparently, the total calculated area of the floor in a grid form has the capability to tell the position of object in the plan with a deviation of $0.02 \mathrm{~m}$ to $0.1 \mathrm{~m}$.

While the potential object was placed at different points in plane then the corresponding IPM frame value is being calculated by changing the orientation of Pepper Head in Yaw and Pitch. In Figs. 5 and 6 it can clearly be seen that by changing head orientaion the actual point is $0.3 \mathrm{~m}$ in $X_{\text {axis }}$ and $1.8 \mathrm{~m}$ in $Y_{\text {axis }}$ as per world frame but the average error in both figure is almost very near to actual values. The average error is due to the camera lens, but it is almost negligible.

The WR has been considered that adopts the path following algorithm as mentioned in (Morro et al., 2011). Fig. 4, validates the Pepper camera and IPM view that shows while the robot is moving in a circular path, the WR position is being calculated depending upon the potential objected tracked that is mounted on the top of roomba.

The Pepper IPM view response while tracking the odometry of Roomba is shown in Fig. 7, further Eq. 9 is used to calculate the average error, that is $a v|e|=0.102 m$. 

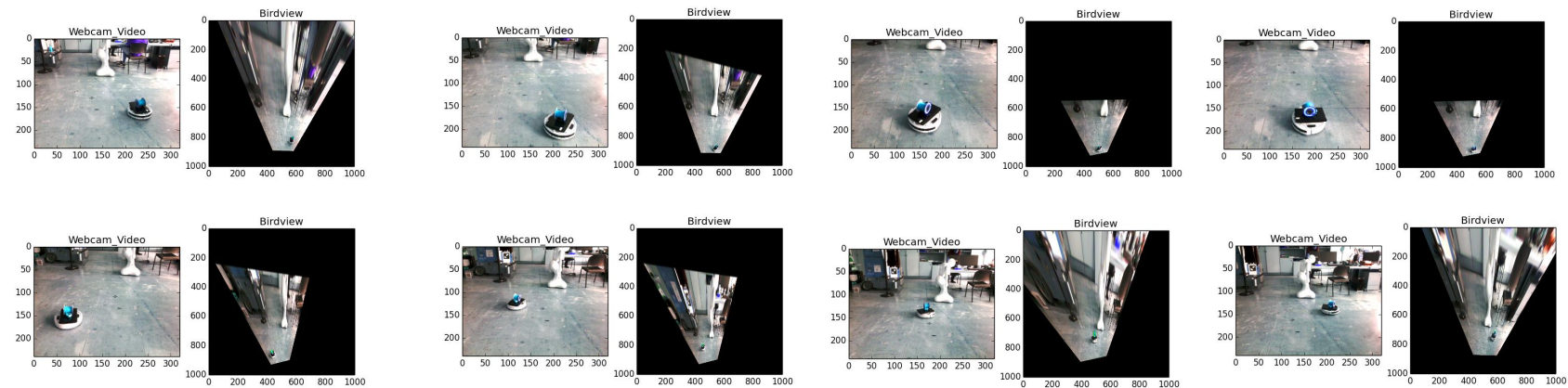

Figure 4: Moving robot in Pepper Monocular camera view and IPM Birdeye-view making a circular path

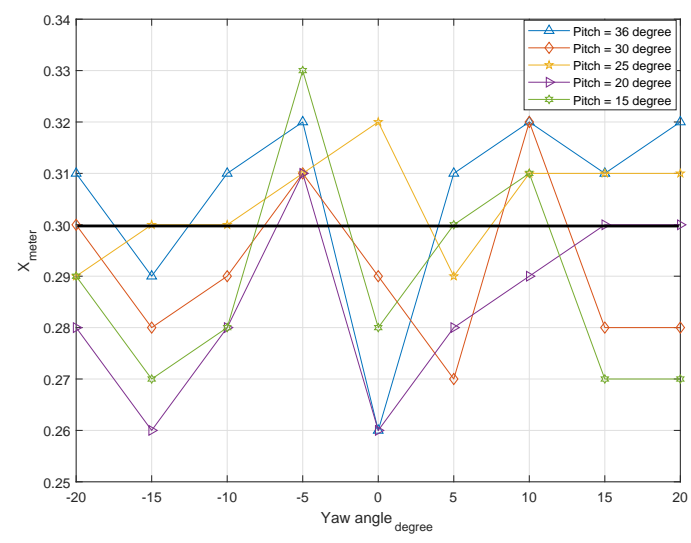

Figure 5: $X_{a x i s}$-IPM view with different Pepper Head Orientation

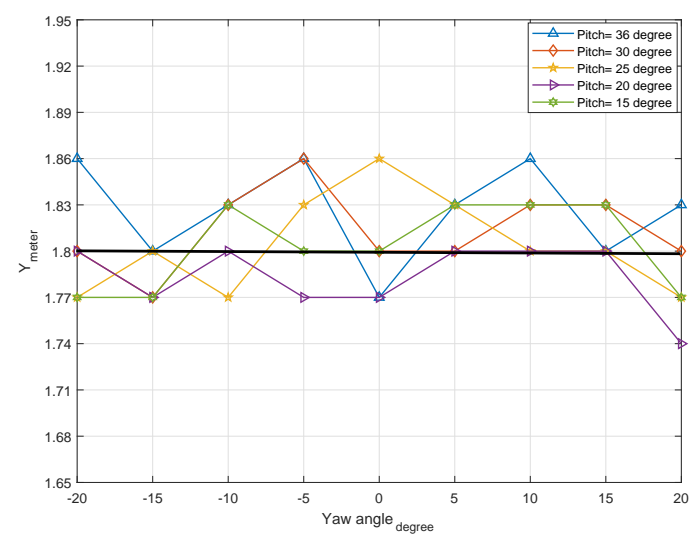

Figure 6: $Y_{a x i s}$-IPM view with different Pepper Head Orientation

\section{CONCLUSION}

This article presents an efficient collaboration method in real-time for Pepper Humanoid robot to know the position of other moving robot or object in the plane. It is mainly based on visual matching and IPM which gives the linear relationship between IPM frame and world frame. So, the experimental results prove that

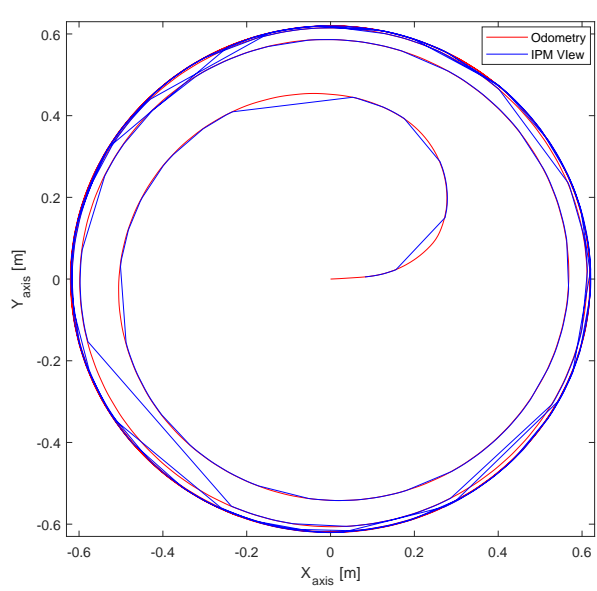

Figure 7: Response of IPM view in a circular path

the position of other potential object is known while the Pepper robot head orientation is static or moving. It is shown in the results and discussion section that the more the number of recording pairs (i.e: Yaw, Pitch) for each point, gives more accurate calculations. The results validates that it can work efficiently for the autonomous diverse environment too. In the future, more detailed plane information and more specific collision strategies will be worked out to provide a more practical system.

\section{ACKNOWLEDGMENTS}

This work has been supported by the European Commission Horizon2020 Research and Innovation Programme under grant agreement No. 737858 


\section{REFERENCES}

Boyle, D. P., Gupta, H. V., and Sorooshian, S. (2000). Toward improved calibration of hydrologic models: Combining the strengths of manual and automatic methods. Water Resources Research, 36(12):36633674.

Civera, J., Davison, A. J., and Montiel, J. M. (2008). Inverse depth parametrization for monocular slam. IEEE transactions on robotics, 24(5):932-945.

Guo, C., Meguro, J.-i., Kojima, Y., and Naito, T. (2014). Automatic lane-level map generation for advanced driver assistance systems using low-cost sensors. In Robotics and Automation (ICRA), 2014 IEEE International Conference on, pages 3975-3982. IEEE.

Hiremath, S. A., Van Der Heijden, G. W., Van Evert, F. K., Stein, A., and Ter Braak, C. J. (2014). Laser range finder model for autonomous navigation of a robot in a maize field using a particle filter. Computers and Electronics in Agriculture, 100:41-50.

Jeong, J. and Kim, A. (2016). Adaptive inverse perspective mapping for lane map generation with slam. In Ubiquitous Robots and Ambient Intelligence (URAI), 2016 13th International Conference on, pages 38-41. IEEE.

Laganiere, R. (2000). Compositing a bird's eye view mosaic. image, 10:3.

Lemaignan, S., Warnier, M., Sisbot, E. A., Clodic, A., and Alami, R. (2017). Artificial cognition for social human-robot interaction: An implementation. Artificial Intelligence, 247:45-69.

Lin, C.-C. and Wang, M.-S. (2012). A vision based topview transformation model for a vehicle parking assistant. Sensors, 12(4):4431-4446.

Ma, L., Yang, X., and Tao, D. (2014). Person reidentification over camera networks using multi-task distance metric learning. IEEE Transactions on Image Processing, 23(8):3656-3670.

Maurino, D. E., Reason, J., Johnston, N., and Lee, R. B. (2017). Beyond aviation human factors: Safety in high technology systems. Routledge.

Miraldo, P. and Araujo, H. (2013). Calibration of smooth camera models. IEEE transactions on pattern analysis and machine intelligence, 35(9):2091-2103.

Moreno, D. and Taubin, G. (2012). Simple, accurate, and robust projector-camera calibration. In $3 D$ Imaging, Modeling, Processing, Visualization and Transmission (3DIMPVT), 2012 Second International Conference on, pages 464-471. IEEE.

Morro, A., Sgorbissa, A., and Zaccaria, R. (2011). Path following for unicycle robots with an arbitrary path curvature. IEEE Transactions on Robotics, 27(5):10161023.

Mukhtar, A., Xia, L., and Tang, T. B. (2015). Vehicle detection techniques for collision avoidance systems: A review. IEEE Transactions on Intelligent Transportation Systems, 16(5):2318-2338.

Munaro, M., Basso, F., and Menegatti, E. (2016). Openptrack: Open source multi-camera calibration and people tracking for rgb-d camera networks. Robotics and Autonomous Systems, 75:525-538.

Oliveira, M., Santos, V., and Sappa, A. D. (2015). Multimodal inverse perspective mapping. Information $\mathrm{Fu}$ sion, 24:108-121.

Rubenstein, M., Cornejo, A., and Nagpal, R. (2014). Programmable self-assembly in a thousand-robot swarm. Science, 345(6198):795-799.

Siciliano, B. and Khatib, O. (2016). Springer handbook of robotics. Springer.

Stein, G., Dagan, E., Mano, O., and Shashua, A. (2017) Collision warning system. US Patent 9,656,607.

Tuohy, S., O'cualain, D., Jones, E., and Glavin, M. (2010). Distance determination for an automobile environment using inverse perspective mapping in opencv.

Van der Walt, S., Schönberger, J. L., Nunez-Iglesias, J., Boulogne, F., Warner, J. D., Yager, N., Gouillart, E., and Yu, T. (2014). scikit-image: image processing in python. PeerJ, 2:e453.

Wang, X. (2013). Intelligent multi-camera video surveillance: A review. Pattern recognition letters, 34(1):319.

Yenikaya, S., Yenikaya, G., and Düven, E. (2013). Keeping the vehicle on the road: A survey on on-road lane detection systems. ACM Computing Surveys (CSUR), 46(1):2. 